models of drug preparation and administration. In hospital A' all drugs were diluted in a minibag under aseptic conditions, while in hospital B', nurses diluted the drug on the wards and administered it through a buretrol device. A cost analysis time \& motion study was performed to find out the cost of each practise model; 11.3 Euro/ unit in hospital A'versus 13 Euro in hospital B'. Hospital A' used PTZ' treatment based on a streaming system \& antibiogram assistance, while in B', use was according to physician approval and a system of switching.

Conclusions This survey has several limitations: the difficulty of accurately reflecting prescribing practises, equipment and patient case mix. Nevertheless, the benchmarking exercise provided valuable data, which can be used to target key areas for cost control and performance.

No conflict of interest.

\section{OHP-012 ASSESSMENT OF UNIFIED INHALATION GUIDANCE DOCUMENTS FOR DIFFERENT INHALERS AND THE INFLUENCE OF AGE ON INHALATION TECHNIOUE}

\section{doi:10.1136/ejhpharm-2013-000276.386}

${ }^{1} \mathrm{R}$ Ono, ${ }^{1} \mathrm{~A}$ Hosomi, ${ }^{2} \mathrm{~T}$ Horie, ${ }^{3} \mathrm{~J}$ Onozato, ${ }^{1} \mathrm{~T}$ Hashita, ${ }^{4} \mathrm{~T}$ Araki, ${ }^{1} \mathrm{~K}$ lizuka, ${ }^{4} \mathrm{~T}$ Nakamura, ${ }^{4} \mathrm{~K}$ Yamamoto, ${ }^{5} \mathrm{~K}$ Dobashi. 'Gunma University Hospital, Pharmacy, Maebashi-shi, Japan; ${ }^{2}$ Maebashi Red Cross Hospital, Respiratory Medicine, Maebashi-shi, Japan; ${ }^{3}$ Maebashi Red Cross Hospital, Pharmacy, Maebashi-shi, Japan; ${ }^{4}$ Gunma University Graduate School of Medicine, Clinical Pharmacology, Maebashi-shi, Japan; ${ }^{5}$ Gunma University Graduate School of Health Sciences, Maebashi-shi, Japan

Background When using an inhaler for asthma or chronic obstructive pulmonary disease, the correct inhalation technique is essential for obtaining the desired effect. However, the development of different types of inhalers has led to inhalation techniques that differ greatly among devices. Therefore, we prepared the 'Unified Inhalation Guidance Documents' (UIGDs) in cooperation with hospitals and pharmacies in our region for each available type of inhaler.

Purpose To assess the benefits and problems of the UIGD.

Materials and Methods A total of 165 Japanese patients who received inhalation therapy from June 2011 to September 2012 were enrolled, and 213 points regarding the inhalation technique with 8 types of inhalers were obtained. The inhalation technique of patients who received guidance based on the UIGD for the use of inhalers was assessed by scoring inhalation skill. In addition, we assessed the impact of age on the acquisition of inhalation technique.

Results We found that 86 cases (40.4\%) showed problems with inhalation technique. In particular, patients using a Breezhaler (4/5) and Turbuhaler (18/37) had a high rate of problems with their technique. Problems were more frequent in patients aged 65 or over (older) $(63 / 146,43.2 \%)$ compared with other patients (younger) $(23 / 67,34.3 \%)$. In particular, for patients using a Turbuhaler, more older patients $(10 / 14,71.4 \%)$ than younger patients $(8 / 23,34.8 \%)$ had trouble with technique.

Conclusions Around $40 \%$ of patients who received guidance based on the UIGD for the use of inhaler devices had trouble with their inhalation technique. Therefore, the UIGDs for the Breezhaler and Turbuhaler should be reassessed. Because the use of some types of inhalers was difficult for older patients, developing an inhaler and guidance specifically for older patients should be considered.

No conflict of interest.

\section{OHP-013 BLEEDING IN CARDIAC SURGERY: USE OF BLOOD COAGULATION FACTORS}

doi:10.1136/ejhpharm-2013-000276.387

V Calmels, A Recurt-Carrere, E Divol, B Sallerin. Chu Toulouse - Rangueil, Pharmacy, Toulouse, France
Background There are few references or publications about the management of bleeding during cardiac surgery. Practices are influenced by the availability of drugs and equipment and are team-dependant.

Off-label use of blood coagulation factors (BCFs) has become a major public health and financial concern.

Purpose To explain the increasing use of BCFs, during cardiac surgery under Extracorporeal Circulation (ECC), in a University Hospital setting.

Materials and Methods We assessed the amounts of BCFs and Transfused Blood Products (TBPs) used between 2009 and 2011 and compared these figures to the number of operations using ECC.

Results During this 2 years, the workload, in number of operations requiring ECC, increased by only $3 \%$ (with a decrease of $11 \%$ in emergencies).

The use of TBP increased $3 \%$.

In the same time, BCF prescriptions increased dramatically (representing a cost of Euro 270,000). The analysis of quantities dispensed shows:

- An increase of over 138\% for Prothrombin Complex Concentrates (PCCs)

- Increase of over $586 \%$ for fibrinogen (Fg)

- Increase of over $102 \%$ for activated factor VII

National figures for the same period were:

- $23 \%$ increase for PCC

- $70 \%$ increase for $\mathrm{Fg}$

- $4 \%$ increase for FVII

Conclusions The increased use of these factors can be explained by changes in local professional practise. In order to standardise and justify the use of these costly products, multidisciplinary meetings (anaesthetists, biologists, cardiac surgeons and pharmacists) are taking place. A procedure for the management of bleeding during cardiac surgery is in development. This document should contribute to the improvement of care in terms of therapeutic efficiency and safety.

No conflict of interest.

\section{OHP-014 CLINICAL AND FINANCIAL EFFECTS OF THE USE OF A THERAPEUTIC EOUIVALENCE PROGRAMME IN A TERTIARY HOSPITAL}

doi:10.1136/ejhpharm-2013-000276.388

'C García, ${ }^{2} \mathrm{C}$ Alarcón, 'I Cañamares, 'I Escribano, 'MJ Jiménez, 'JM Ferrari. 'Hospital Universitario 12 de Octubre, Pharmacy, Madrid, Spain; ${ }^{2}$ Hospital Universitario Virgen de las Nieves, Pharmacy, Granada, Spain

Background A Therapeutic Equivalence Program (TEP) assembles clinically equivalent drugs and defines the best therapeutic alternative included in the hospital's pharmacotherapeutic guide to drugs not included (DNI).

Purpose To measure the clinical and financial impact of a TEP in a tertiary hospital.

Materials and Methods Descriptive observational study conducted between November 2011 and January 2012. During the transcription and validation of the prescriptions of clinical units that work with unit-dose drug distribution system, pharmacist applied the TEP and notified the physician of the substitution in writing. The prescription of an unincluded was only retained if there was a clinical justification that made substitution impossible.

The variables collected were: rate of substitution proposals accepted and rejected, justifications for not performing the substitution, cost of the DNI, cost of the therapeutic equivalent per hospital stay and percentage of therapeutic equivalents prescribed at discharge. 
Results 199 substitution proposals were sent to the physicians (51.8\% accepted, $48.2 \%$ not accepted. Of these, in $17.1 \%$ of cases the patient brought the medicine from home and in $7 \%$ treatment was discontinued).

The most common clinical justification accepted (8 cases) was leg oedema caused by amlodipine (maintenance of manidipine). The second one was anaerobic infection where levofloxacin is not active (maintenance of moxifloxacin).

The global DNI price within two months of study was $€ 1,148.78$. The cost saving with the acceptance of $51.8 \%$ of substitutions was $€ 472.63$ in two months. If $100 \%$ of substitutions had been accepted, the therapeutic equivalent prescription would have saved $€ 586.75$.

In $17 \%$ of cases therapeutic equivalents were prescribed at discharge.

Conclusions The suggested substitution was accepted in more than half of cases.

The adjustment of medical prescriptions to the hospital's pharmacotherapeutic guide prevailed over the economic saving, which was not significant.

The prescription of therapeutic equivalents at discharge was not as expected.

No conflict of interest.

\section{OHP-015 CLINICAL RESEARCH IN FRANCE AND QUEBEC}

doi:10.1136/ejhpharm-2013-000276.389

'A Guérin, ' $\mathrm{C}$ Tanguay, 'D Lebel, ${ }^{2} \mathrm{O}$ Bourdon, ${ }^{1 J F}$ Bussières. ${ }^{1} \mathrm{CHU}$ Sainte-Justine, Pharmacy, Montreal, Canada; ${ }^{2}$ Hôpital Universitaire Robert-Debré, Pharmacy, Paris, France

Background Pharmacy practise is evolving in most countries. Hospital pharmacists are pivotal in the organisation and the support of clinical trials. We looked at the current state of pharmacy practise in clinical research

Purpose To identify differences in clinical research organisation and pharmacy practise between France and Quebec (Canada).

Materials and Methods This is a descriptive study. A literature review was performed in order to describe the organisation of clinical research and the role of pharmacists in clinical research for both countries. Differences were identified by a panel consisting of one French pharmacy intern, one French hospital pharmacist, one Quebec research assistant and two Quebec hospital pharmacists.

Results Fourteen differences relating to research organisation were identified. France and Canada have different normative frameworks, regulatory authorities, authorization processes, delays and shutdown processes. While it is encouraged, clinical trial registration is not mandatory in Canada. Data needs to be archived for 15 years in France vs. 25 years in Canada. Institutional review boards (IRB) have different names, location, composition, nomination processes, mandate duration and informed consent processes for minors. Seven key differences in pharmacy practise were identified. There are different authorization processes for drug compounding and manufacturing. Pharmacy fees are based on a national reference in France, but not in Canada. Software for the computerization of pharmacy services for clinical trials is common in France. In addition to drug trials, French pharmacists also manage sterile medical devices and medicinal products derived from human blood. Canadian pharmacists offer decentralised pharmaceutical care to hospitalised patients. Canadian pharmacists can be principal investigators if a doctor is the qualified investigator.

Conclusions Clinical research organisation is similar on many aspects, but 21 main differences were identified. Comparisons between countries help identify best practise and may contribute to practise improvement.

No conflict of interest.

\section{OHP-016 CONSUMPTION OF OPIOID ANALGESICS IN HOSPITAL PHARMACY AND CONSULTATIVE CARE FOR PATIENTS}

doi:10.1136/ejhpharm-2013-000276.390

1J Sumberova, ${ }^{2} \mathrm{M}$ Lzicar, ${ }^{3} \mathrm{~J}$ Kolar. 'Pharmacy Konstancie, Community Pharmacy, Brno, Czech Republic; '2St. Ann's University Hospital, Hospital Pharmacy, Brno, Czech Republic; ${ }^{3}$ University of Veterinary and Pharmaceutical Sciences, Faculty of Pharmacy, Brno, Czech Republic

Background In recent years the incidence of different types of pain is increasing. We have found the same in the St. Anne's hospital in the Czech Republic. Patients are now able to ask about the correct usage of opioid drugs in pharmacy consultation centre, which opened in 2011.

Purpose To find out the consumption of opioid analgesics from 2008 to 2011. This is an analysis of prescriptions by doctors from the pain treatment centre. We also collected data from patient records in the pharmacy consultation centre and we wanted to know how many patients come to consult us.

Materials and Methods Data were obtained from the pharmacy computer software. We made a retrospective evaluation, calculated the defined daily dosage (DDD) and compared consumption of opioid analgesics during 2008-2011 for ATC class N02A and other subclasses. We analysed the consultation records.

Results Consumption of weak opioids decreased over that time, while consumption of strong opioids increased, which had to be prescribed. Opiates were prescribed more often to women. The highest consumption was of buprenorphine, than fentanyl and oxycodone, from weak opioids it was tramadol. Consumption of fentanyl increased from 35735 DDD (2010) to 39924 DDD (2011), while buprenorphine consumption decreased from 45059 DDD (2010) to 38675 DDD (2011). The amount of morphine used last year was twice that of previous years. The total number of patients who visited the pharmacy consulting centre was 41 , six patients were from the pain treatment centre. Average age was 61.3. Patients wanted to control interactions, secondly combat adverse effects of opioids and requested information about neuropathic pain. Average consultation length was 22.5 minutes.

Conclusions The consumption of strong opioids is gradually increasing, doctors follow guidelines and they aren't afraid of prescribing strong opioids. In future it would be appropriate to extend the distribution of informatory materials by the consultation centre - not only about the opioid analgesics.

No conflict of interest.

\section{OHP-017 COST COMPARISON OF INTRAVITREAL ANTIANGIOGENIC DRUGS IN THE TREATMENT OF AGE-RELATED MACULAR DEGENERATION}

doi:10.1136/ejhpharm-2013-000276.391

'C Matallana, 'MC Izquierdo, 'V Martinez, 'M Hernando, ${ }^{2 P}$ Aznarte, ${ }^{3} \mathrm{MT}$ Sanchez. ${ }^{1}$ Hospital Clínico Universitario, Pharmacy, Valladolid, Spain; ${ }^{2}$ Hospital Virgen de las Nieves, Pharmacy, Granada, Spain; ${ }^{3}$ Hospital Clinico Universitario, Pharmacy, Valladolid, Spain

Background The primary treatment of Age-related Macular Degeneration (AMD) is based on inhibition of Vascular Epithelial Growth Factor (VEGF) with antiangiogenic drugs, which delay disease progression and improve the patient's vision.

Choosing between bevacizumab and ranibizumab is still up for debate. Bevacizumab has not been approved for $\mathrm{AMD}$, while ranibizumab has a safer profile and is legally approved for this condition, although it is more expensive.

Purpose To evaluate the cost of intravitreal ranibizumab in AMD and to compare with the hypothetical cost of treatment with intravitreal bevacizumab in off-label conditions for the same group of patients. 